\title{
Lower Bound of Concurrence for Multipartite Quantum States
}

\author{
Ming Li ${ }^{1}$, Shao-Ming Fei ${ }^{1,2}$ and Zhi-Xi Wang ${ }^{1}$ \\ ${ }^{1}$ Department of Mathematics, Capital Normal University, Beijing 100037 \\ ${ }^{2}$ Institut für Angewandte Mathematik, Universität Bonn, D-53115
}

\begin{abstract}
We study the concurrence of arbitrary multipartite mixed quantum states. An explicit lower bound of the concurrence is derived, which detects quantum entanglement of some states better than some separability criteria, and gives sufficient conditions for distilling GHZ states from tripartite states. An interesting relations between the lower bound of the concurrence for bipartite states and for tripartite states has been presented.

PACS numbers: 03.67.-a, 02.20.Hj, 03.65.-w
\end{abstract}

\section{Introduction}

Quantum entanglement plays crucial roles in quantum information processing [1]. Entanglement of formation (EOF) [2] and concurrence [3, 4] are two well defined quantitative measures of quantum entanglement. For two-quibt systems it has been proved that EOF is a monotonically increasing function of the concurrence and an elegant formula for the concurrence was derived analytically by Wootters [5]. However with the increasing dimensions of the subsystems the computation of EOF and concurrence become formidably difficult. A few explicit analytic formulae for EOF and concurrence have been found only for some special symmetric states $[6,7,8,9,10]$.

The first analytic lower bound of concurrence that can be tightened by numerical optimization over some parameters was derived in [11]. In $[12,13]$ analytic lower bounds on EOF and concurrence for any dimensional mixed bipartite quantum states have been presented by using the positive partial transposition (PPT) and realignment separability criteria. These bounds are exact for some special classes of states and can be used to detect many bound entangled states. In [14] another lower bound on EOF for bipartite states has been presented from a new separability criterion [15]. A lower bound of concurrence based on local uncertainty relations (LURs) criterion is derived in [16]. This bound is further optimized in [17]. The lower bound of concurrence for tripartite systems has been studied in [18].

In $[19,20]$ the authors presented lower bounds of concurrence for bipartite systems in terms of a different approach. It has been shown that this lower bound has a close relationship with the distillability of bipartite quantum states. 
In this letter, we study the lower bound of concurrence for arbitrary multipartite quantum systems by using the approach in [20]. Let $H$ denotes a $d$-dimensional vector space with basis $|i\rangle, i=1,2, \ldots, d$. An $N$-partite pure state in $H \otimes \cdots \otimes H$ is generally of the form,

$$
|\Psi\rangle=\sum_{i_{1}, i_{2}, \cdots i_{N}=1}^{d} a_{i_{1}, i_{2}, \cdots i_{N}}\left|i_{1}, i_{2}, \cdots i_{N}\right\rangle, \quad a_{i_{1}, i_{2}, \cdots i_{N}} \in \mathbb{C} .
$$

Let $\alpha$ and $\alpha^{\prime}$ (resp. $\beta$ and $\beta^{\prime}$ ) be subsets of the subindices of $a$, associated to the same sub Hilbert spaces but with different summing indices. $\alpha$ (or $\alpha^{\prime}$ ) and $\beta$ (or $\beta^{\prime}$ ) span the whole space of the given sub-indix of $a$. The generalized concurrence of $|\Psi\rangle$ is then given by $[4]$

$$
C_{d}^{N}(|\Psi\rangle)=\sqrt{\frac{d}{2 m(d-1)} \sum_{p} \sum_{\left\{\alpha, \alpha^{\prime}, \beta, \beta^{\prime}\right\}}^{d}\left|a_{\alpha \beta} a_{\alpha^{\prime} \beta^{\prime}}-a_{\alpha \beta^{\prime}} a_{\alpha^{\prime} \beta}\right|^{2}},
$$

where $m=2^{N-1}-1, \sum_{p}$ stands for the summation over all possible combinations of the indices of $\alpha$ and $\beta$.

For a mixed state $\rho$,

$$
\rho=\sum_{i} p_{i}\left|\psi_{i}\right\rangle\left\langle\psi_{i}\right|, \quad p_{i} \geq 0, \quad \sum_{i} p_{i}=1
$$

the concurrence is defined by the convex-roof:

$$
C(\rho)=\min \sum_{i} p_{i} C\left(\left|\psi_{i}\right\rangle\right)
$$

minimized over all possible pure state decompositions.

\section{Lower bound of the concurrence of a multipartite quantum state}

We first consider tripartite case. A general pure state on $H \otimes H \otimes H$ is of the form

$$
|\Psi\rangle=\sum_{i, j, k=1}^{d} a_{i j k}|i j k\rangle, \quad a_{i j k} \in \mathbb{C}, \quad \sum_{i, j, k=1}^{d} a_{i j k} a_{i j k}^{*}=1
$$

with

$C_{d}^{3}(|\Psi\rangle)=\sqrt{\frac{d}{6(d-1)} \sum\left(\left|a_{i j k} a_{p q m}-a_{i j m} a_{p q k}\right|^{2}+\left|a_{i j k} a_{p q m}-a_{i q k} a_{p j m}\right|^{2}+\left|a_{i j k} a_{p q m}-a_{p j k} a_{i q m}\right|^{2}\right)}$

or equivalently

$$
C_{d}^{3}(|\Psi\rangle)=\sqrt{\frac{d}{6(d-1)}\left(3-\left(\operatorname{Tr} \rho_{1}^{2}+\operatorname{Tr} \rho_{2}^{2}+\operatorname{Tr} \rho_{3}^{2}\right)\right)},
$$


where $\rho_{1}=\operatorname{Tr}_{23}(\rho), \rho_{2}=\operatorname{Tr}_{13}(\rho), \rho_{3}=\operatorname{Tr}_{12}(\rho)$ are the reduced density matrices of $\rho=$ $|\Psi\rangle\langle\Psi|$.

Define

$$
\begin{aligned}
& C_{\alpha \beta}^{12 \mid 3}(|\Psi\rangle)=\left|a_{i j k} a_{p q m}-a_{i j m} a_{p q k}\right|, \quad C_{\alpha \beta}^{13 \mid 2}(|\Psi\rangle)=\left|a_{i j k} a_{p q m}-a_{i q k} a_{p j m}\right|, \\
& C_{\alpha \beta}^{23 \mid 1}(|\Psi\rangle)=\left|a_{i j k} a_{p q m}-a_{p j k} a_{i q m}\right|,
\end{aligned}
$$

where $\alpha$ and $\beta$ of $C_{\alpha \beta}^{12 \mid 3}$ (resp. $C_{\alpha \beta}^{13 \mid 2}$ resp. $C_{\alpha \beta}^{23 \mid 1}$ ) stand for the sub-indices of $a$ associated with the subspaces 1,2 and 3 (resp. 1,3 and 2 resp. 2,3 and 1). Let $L^{i_{1} i_{2} \cdots i_{N}}$ denote the generators of group $S O\left(d_{i_{1}} d_{i_{2}} \cdots d_{i_{N}}\right)$ associated to the subsystems $i_{1}, i_{2}, \cdots, i_{N}$. Then for a tripartite pure state (5), one has

$$
\begin{aligned}
C_{d}^{3}(|\Psi\rangle) & =\sqrt{\frac{d}{6(d-1)} \sum_{\alpha}^{\frac{d^{2}\left(d^{2}-1\right)}{2}} \frac{d(d-1)}{2} \sum_{\beta}^{2}\left[\left(C_{\alpha \beta}^{12 \mid 3}(|\Psi\rangle)\right)^{2}+\left(C_{\alpha \beta}^{13 \mid 2}(|\Psi\rangle)\right)^{2}+\left(C_{\alpha \beta}^{23 \mid 1}(|\Psi\rangle)\right)^{2}\right]} \\
& =\sqrt{\frac{d}{6(d-1)} \sum_{\alpha \beta}\left[\left(\left|\left\langle\Psi\left|S_{\alpha \beta}^{12 \mid 3}\right| \Psi^{*}\right\rangle\right|\right)^{2}+\left(\left|\left\langle\Psi\left|S_{\alpha \beta}^{13 \mid 2}\right| \Psi^{*}\right\rangle\right|\right)^{2}+\left(\left|\left\langle\Psi\left|S_{\alpha \beta}^{23 \mid 1}\right| \Psi^{*}\right\rangle\right|\right)^{2}\right]},
\end{aligned}
$$

where $S_{\alpha \beta}^{12 \mid 3}=\left(L_{\alpha}^{12} \otimes L_{\beta}^{3}\right), S_{\alpha \beta}^{13 \mid 2}=\left(L_{\alpha}^{13} \otimes L_{\beta}^{2}\right)$ and $S_{\alpha \beta}^{23 \mid 1}=\left(L_{\beta}^{1} \otimes L_{\alpha}^{23}\right)$.

Theorem 1: For an arbitrary mixed state $(3)$ in $H \otimes H \otimes H$, the concurrence $C(\rho)$ satisfies

$$
\tau_{3}(\rho) \equiv \frac{d}{6(d-1)} \sum_{\alpha}^{\frac{d^{2}\left(d^{2}-1\right)}{2}} \sum_{\beta}^{\frac{d(d-1)}{2}}\left[\left(C_{\alpha \beta}^{12 \mid 3}(\rho)\right)^{2}+\left(C_{\alpha \beta}^{13 \mid 2}(\rho)\right)^{2}+\left(C_{\alpha \beta}^{23 \mid 1}(\rho)\right)^{2}\right] \leq C^{2}(\rho),
$$

where $\tau_{3}(\rho)$ is a lower bound of $C(\rho)$,

$$
C_{\alpha \beta}^{12 \mid 3}(\rho)=\max \left\{0, \lambda(1)_{\alpha \beta}^{12 \mid 3}-\lambda(2)_{\alpha \beta}^{12 \mid 3}-\lambda(3)_{\alpha \beta}^{12 \mid 3}-\lambda(4)_{\alpha \beta}^{12 \mid 3}\right\}
$$

$\lambda(1)_{\alpha \beta}^{12 \mid 3}, \lambda(2)_{\alpha \beta}^{12 \mid 3}, \lambda(3)_{\alpha \beta}^{12 \mid 3}, \lambda(4)_{\alpha \beta}^{12 \mid 3}$ are the square roots of the four nonzero eigenvalues, in decreasing order, of the non-Hermitian matrix $\rho \widetilde{\rho}_{\alpha \beta}^{12 \mid 3}$ with $\tilde{\rho}_{\alpha \beta}^{12 \mid 3}=S_{\alpha \beta}^{12 \mid 3} \rho^{*} S_{\alpha \beta}^{12 \mid 3} . C_{\alpha \beta}^{13 \mid 2}(\rho)$ and $C_{\alpha \beta}^{23 \mid 1}(\rho)$ are defined in a similar way to $C_{\alpha \beta}^{12 \mid 3}(\rho)$.

Proof: Set $\left|\xi_{i}\right\rangle=\sqrt{p_{i}}\left|\psi_{i}\right\rangle, x_{\alpha \beta}^{i}=\left|\left\langle\xi_{i}\left|S_{\alpha \beta}^{12 \mid 3}\right| \xi_{i}^{*}\right\rangle\right|, y_{\alpha \beta}^{i}=\left|\left\langle\xi_{i}\left|S_{\alpha \beta}^{13 \mid 2}\right| \xi_{i}^{*}\right\rangle\right|$ and $z_{\alpha \beta}^{i}=\left|\left\langle\xi_{i}\left|S_{\alpha \beta}^{1 \mid 23}\right| \xi_{i}^{*}\right\rangle\right|$. We have, from Minkowski inequality

$$
\begin{aligned}
C(\rho) & =\min \sum_{i} \sqrt{\frac{d}{6(d-1)} \sum_{\alpha \beta}\left[\left(x_{\alpha \beta}^{i}\right)^{2}+\left(y_{\alpha \beta}^{i}\right)^{2}+\left(z_{\alpha \beta}^{i}\right)^{2}\right]} \\
& \geq \min \sqrt{\frac{d}{6(d-1)} \sum_{\alpha \beta}\left(\sum_{i}\left[\left(x_{\alpha \beta}^{i}\right)^{2}+\left(y_{\alpha \beta}^{i}\right)^{2}+\left(z_{\alpha \beta}^{i}\right)^{2}\right]^{\frac{1}{2}}\right)^{2}} .
\end{aligned}
$$

Noting that for nonnegative real variables $x_{\alpha}, y_{\alpha}, z_{\alpha}$ and given $X=\sum_{\alpha=1}^{N} x_{\alpha}, Y=\sum_{\alpha=1}^{N} Y_{\alpha}$ and $Z=\sum_{\alpha=1}^{N} z_{\alpha}$, by using Lagrange multipliers one obtain that the following inequality 
holds,

$$
\sum_{\alpha=1}^{N}\left(x_{\alpha}^{2}+y_{\alpha}^{2}+z_{\alpha}^{2}\right)^{\frac{1}{2}} \geq\left(X^{2}+Y^{2}+Z^{2}\right)^{\frac{1}{2}} .
$$

Therefore we have

$$
\begin{aligned}
C(\rho) & \geq \min \sqrt{\frac{d}{6(d-1)} \sum_{\alpha \beta}\left[\left(\sum_{i} x_{\alpha \beta}^{i}\right)^{2}+\left(\sum_{i} y_{\alpha \beta}^{i}\right)^{2}+\left(\sum_{i} z_{\alpha \beta}^{i}\right)^{2}\right]} \\
& \geq \sqrt{\frac{d}{6(d-1)} \sum_{\alpha \beta}\left[\left(\min \sum_{i} x_{\alpha \beta}^{i}\right)^{2}+\left(\min \sum_{i} y_{\alpha \beta}^{i}\right)^{2}+\left(\min \sum_{i} z_{\alpha \beta}^{i}\right)^{2}\right]} .
\end{aligned}
$$

The values of $C_{\alpha \beta}^{12 \mid 3}(\rho) \equiv \min \sum_{i} x_{\alpha \beta}^{i}, C_{\alpha \beta}^{13 \mid 2}(\rho) \equiv \min \sum_{i} y_{\alpha \beta}^{i}$ and $C_{\alpha \beta}^{23 \mid 1}(\rho) \equiv \min \sum_{i} z_{\alpha \beta}^{i}$ can be calculated by using the similar procedure in [5]. Here we compute the value of $C_{\alpha \beta}^{12 \mid 3}(\rho)$ in detail. The values of $C_{\alpha \beta}^{13 \mid 2}(\rho)$ and $C_{\alpha \beta}^{23 \mid 1}(\rho)$ can be obtained analogously.

Let $\lambda_{i}$ and $\left|\chi_{i}\right\rangle$ be eigenvalues and eigenvectors of $\rho$ respectively. Any decomposition of $\rho$ can be obtained from a unitary $d^{3} \times d^{3} \operatorname{matrix} V_{i j},\left|\xi_{j}\right\rangle=\sum_{i=1}^{d^{3}} V_{i j}^{*}\left(\sqrt{\lambda_{i}}\left|\chi_{i}\right\rangle\right)$. Therefore one has $\left\langle\xi_{i}\left|S_{\alpha \beta}^{12 \mid 3}\right| \xi_{j}^{*}\right\rangle=\left(V Y_{\alpha \beta} V^{T}\right)_{i j}$, where the matrix $Y_{\alpha \beta}$ is defined by $\left(Y_{\alpha \beta}\right)_{i j}=\left\langle\chi_{i}\left|S_{\alpha \beta}^{12 \mid 3}\right| \chi_{j}^{*}\right\rangle$. Namely $C_{\alpha \beta}^{12 \mid 3}(\rho)=\min \sum_{i}\left|\left[V Y_{\alpha \beta} V^{T}\right]_{i i}\right|$, which has an analytical expression [5], $C_{\alpha \beta}^{12 \mid 3}(\rho)=$ $\max \left\{0, \lambda(1)_{\alpha \beta}^{12 \mid 3}-\sum_{j>1} \lambda(j)_{\alpha \beta}^{12 \mid 3}\right\}$, where $\lambda_{\alpha \beta}^{12 \mid 3}(k)$ are the square roots of the eigenvalues of the positive Hermitian matrix $Y_{\alpha \beta} Y_{\alpha \beta}^{\dagger}$, or equivalently the non-Hermitian matrix $\rho \widetilde{\rho}_{\alpha \beta}$, in decreasing order. Here as the matrix $S_{\alpha \beta}^{12 \mid 3}$ has $d^{2}-4$ rows and $d^{2}-4$ columns that are identically zero, the matrix $\rho \widetilde{\rho}_{\alpha \beta}$ has a rank no greater than 4 , i.e., $\lambda_{\alpha \beta}^{12 \mid 3}(j)=0$ for $j \geq 5$. From Eq.(13) we have Eq.(10).

Theorem 1 can be directly generalized to arbitrary multipartite case.

Theorem 2: For an arbitrary $N$-partite state $\rho \in H \otimes H \otimes \cdots \otimes H$, the concurrence defined in (4) satisfies:

$$
\tau_{N}(\rho) \equiv \frac{d}{2 m(d-1)} \sum_{p} \sum_{\alpha \beta}\left(C_{\alpha \beta}^{p}(\rho)\right)^{2} \leq C^{2}(\rho),
$$

where $\tau_{N}(\rho)$ is the lower bound of $C(\rho), \sum_{p}$ stands for the summation over all possible combinations of the indices of $\alpha, \beta, C_{\alpha \beta}^{p}(\rho)=\max \left\{0, \lambda(1)_{\alpha \beta}^{p}-\lambda(2)_{\alpha \beta}^{p}-\lambda(3)_{\alpha \beta}^{p}-\lambda(4)_{\alpha \beta}^{p}\right\}$, $\lambda(i)_{\alpha \beta}^{p}, i=1,2,3,4$, are the square roots of the four nonzero eigenvalues, in decreasing order, of the non-Hermitian matrix $\rho \widetilde{\rho}_{\alpha \beta}^{p}$ where $\widetilde{\rho}_{\alpha \beta}^{p}=S_{\alpha \beta}^{p} \rho^{*} S_{\alpha \beta}^{p}$.

\section{The lower bound and separability}

An N-partite quantum state $\rho$ is fully separable if and only if there exist $p_{i}$ with $p_{i} \geq$ $0, \sum_{i} p_{i}=1$ and pure states $\rho_{i}^{j}=\left|\psi_{i}^{j}\right\rangle\left\langle\psi_{i}^{j}\right|$ such that

$$
\rho=\sum_{i} p_{i} \rho_{i}^{1} \otimes \rho_{i}^{2} \otimes \cdots \otimes \rho_{i}^{N}
$$


It is easily verified that for a fully separable multipartite state $\rho, \tau_{N}(\rho)=0$. Thus $\tau_{N}(\rho)>0$ indicates that there must be some kinds of entanglement inside the quantum state, which shows that the lower bound $\tau_{N}(\rho)$ can be used to recognize entanglement.

As an example we consider a tripartite quantum state [21], $\rho=\frac{1-p}{8} I_{8}+p|W\rangle\langle W|$, where $I_{8}$ is the $8 \times 8$ identity matrix, and $|W\rangle=\frac{1}{\sqrt{3}}(|100\rangle+|010\rangle+|001\rangle)$ is the tripartite Wstate. Select an entanglement witness operator to be $\mathcal{W}=\frac{1}{2} I_{8}-|G H Z\rangle\langle G H Z|$, where $|G H Z\rangle=\frac{1}{\sqrt{2}}(|000\rangle+|111\rangle)$ to be the tripartite GHZ-state. By computing $\operatorname{Tr}\{\mathcal{W} \rho\}<0$ the entanglement of $\rho$ is detected for $\frac{3}{5}<p \leq 1$ in [21]. In [22] the authors have obtained the generalized correlation matrix criterion which says if an N-qubit quantum state is fully separable then the inequality $\left\|\mathcal{T}^{N}\right\|_{K F} \leq 1$ must hold, where $\left\|\mathcal{T}^{N}\right\|_{K F}=\max \left\{\left\|\mathcal{T}_{n}^{N}\right\|_{K F}\right\}$, $\mathcal{T}_{n}^{N}$ is a kind of matrix unfold of $t_{\alpha_{1} \alpha_{2} \cdots \alpha_{N}}$ defined by $t_{\alpha_{1} \alpha_{2} \cdots \alpha_{N}}=\operatorname{Tr}\left\{\rho \sigma_{\alpha_{1}}^{(1)} \sigma_{\alpha_{2}}^{(2)} \cdots \sigma_{\alpha_{N}}^{(N)}\right\}$ and $\sigma_{\alpha_{i}}^{(i)}$ stands for the pauli matrix. Now using the generalized correlation matrix criterion the entanglement of $\rho$ is detected for $0.3068<p \leq 1$. From our theorem, we have that the lower bound $\tau_{3}(\rho)>0$ for $0.2727<p \leq 1$. Therefore our bound detects entanglement better than these two criteria in this case. If we replace $\mathrm{W}$ with GHZ state in $\rho$, the criterion in [22] detects the entanglement of $\rho$ for $0.35355<p \leq 1$, while $\tau_{3}(\rho)$ detects, again better, the entanglement for $0.2<p \leq 1$.

Nevertheless for PPT states $\rho$, we have $\tau_{3}(\rho)=0$, which can be seen in the following way. A density matrix $\rho$ is called PPT if the partial transposition of $\rho$ over any subsystem(s) is still positive. Let $\rho^{T_{i}}$ denote the partial transposition with respect to the $i$-th subsystem. Assume that there is a PPT state $\rho$ with $\tau(\rho)>0$. Then at least one term in (10), say

$C_{\alpha_{0} \beta_{0}}^{12 \mid 3}(\rho)$, is not zero. Define $\rho_{\alpha_{0} \beta_{0}}=L_{\alpha_{0}}^{12} \otimes L_{\beta_{0}}^{3} \rho\left(L_{\alpha_{0}}^{12} \otimes L_{\beta_{0}}^{3}\right)^{\dagger}$. By using the PPT property of $\rho$, we have:

$$
\rho_{\alpha_{0} \beta_{0}}^{T_{3}}=L_{\alpha_{0}}^{12} \otimes\left(L_{\beta_{0}}^{3}\right)^{*} \rho^{T_{3}}\left(L_{\alpha_{0}}^{12}\right)^{\dagger} \otimes\left(L_{\beta_{0}}^{3}\right)^{T} \geq 0 .
$$

Noting that both $L_{\alpha_{0}}^{12}$ and $L_{\beta_{0}}^{3}$ are projectors to two-dimensional subsystems, $\rho_{\alpha_{0} \beta_{0}}$ can be considered as a $4 \times 4$ density matrix. While a PPT $4 \times 4$ density matrix $\rho_{\alpha_{0} \beta_{0}}$ must be a separable state, which contradicts with $C_{\alpha_{0} \beta_{0}}^{12 \mid 3}(\rho) \neq 0$.

\section{Comparison with the lower bound of the bipartite concurrence}

The lower bound $\tau_{2}$ of concurrence for bipartite states has been obtained in [20]. For a bipartite quantum state $\rho$ in $H \otimes H$, the concurrence $C(\rho)$ satisfies

$$
\tau_{2}(\rho) \equiv \frac{d}{2(d-1)} \sum_{m, n=1}^{\frac{d(d-1)}{2}} C_{m n}^{2}(\rho) \leq C^{2}(\rho),
$$

where $C_{m n}^{2}(\rho)=\max \left\{0, \lambda_{m n}(1)-\lambda_{m n}(2)-\lambda_{m n}(3)-\lambda_{m n}(4)\right\}$ with $\lambda_{m n}(1), \ldots, \lambda_{m n}(4)$ being the square roots of the four nonzero eigenvalues, in decreasing order, of the non-Hermitian 
matrix $\rho \widetilde{\rho}_{m n}$ with $\widetilde{\rho}_{m n}=\left(L_{m} \otimes L_{n}\right) \rho^{*}\left(L_{m} \otimes L_{n}\right), L_{m}$ and $L_{n}$ being the generators of $S O(d) . \tau_{3}$ is basically different from $\tau_{2}$ as $\tau_{3}$ characterizes also genuine tripartite entanglement that can not be described by bipartite decompositions. Nevertheless, there are interesting relations between them.

Theorem 3: For any pure tripartite state (5), the following inequality holds:

$$
\tau_{2}\left(\rho_{12}\right)+\tau_{2}\left(\rho_{13}\right)+\tau_{2}\left(\rho_{23}\right) \leq 3 \tau_{3}(\rho),
$$

where $\tau_{2}$ is the lower bound of bipartite concurrence (17), $\tau_{3}$ is the lower bound of tripartite concurrence (10) and $\rho_{12}=\operatorname{Tr}_{3}(\rho), \rho_{13}=\operatorname{Tr}_{2}(\rho), \rho_{23}=\operatorname{Tr}_{1}(\rho), \rho=|\Psi\rangle_{123}\langle\Psi|$.

Proof: Since $C_{\alpha \beta}^{2} \leq\left(\lambda_{\alpha \beta}(1)\right)^{2} \leq \sum_{i=1}^{4}\left(\lambda_{\alpha \beta}(i)\right)^{2}=\operatorname{Tr}\left(\rho \widetilde{\rho}_{\alpha \beta}\right)$ for $\rho=\rho_{12}, \rho=\rho_{13}$ and $\rho=\rho_{23}$, we have

$$
\begin{aligned}
& \tau_{2}\left(\rho_{12}\right)+\tau_{2}\left(\rho_{13}\right)+\tau_{2}\left(\rho_{23}\right) \\
\leq & \frac{d}{2(d-1)}\left(\sum_{\alpha, \beta=1}^{\frac{d(d-1)}{2}} \operatorname{Tr}\left(\rho_{12}\left(\widetilde{\rho}_{12}\right)_{\alpha \beta}\right)+\sum_{\alpha, \beta=1}^{\frac{d(d-1)}{2}} \operatorname{Tr}\left(\rho_{13}\left(\widetilde{\rho}_{13}\right)_{\alpha \beta}\right)+\sum_{\alpha, \beta=1}^{\frac{d(d-1)}{2}} \operatorname{Tr}\left(\rho_{23}\left(\widetilde{\rho}_{23}\right)_{\alpha \beta}\right)\right) \\
= & \frac{d}{2(d-1)}\left(3-\operatorname{Tr} \rho_{1}^{2}-\operatorname{Tr} \rho_{2}^{2}-\operatorname{Tr} \rho_{3}^{2}\right)=3 C^{2}(\rho)=3 \tau_{3}(\rho),
\end{aligned}
$$

where we have used the similar analysis in $[20,25]$ to obtain the equality $\sum_{\alpha, \beta} \operatorname{Tr}\left(\rho_{12}\left(\widetilde{\rho}_{12}\right)_{\alpha \beta}\right)=$ $1-\operatorname{Tr} \rho_{1}^{2}-\operatorname{Tr} \rho_{2}^{2}+\operatorname{Tr} \rho_{3}^{2}, \sum_{\alpha, \beta} \operatorname{Tr}\left(\rho_{13}\left(\widetilde{\rho}_{13}\right)_{\alpha \beta}\right)=1-\operatorname{Tr} \rho_{1}^{2}+\operatorname{Tr} \rho_{2}^{2}-\operatorname{Tr} \rho_{3}^{2}, \sum_{\alpha, \beta} \operatorname{Tr}\left(\rho_{23}\left(\widetilde{\rho}_{23}\right)_{\alpha \beta}\right)=$ $1+\operatorname{Tr} \rho_{1}^{2}-\operatorname{Tr} \rho_{2}^{2}-\operatorname{Tr} \rho_{3}^{2}$. The last equality is due to that $\rho$ is a pure state.

In fact, the bipartite entanglement inside a tripartite state is useful for distilling maximally entangled states. Assume that there are two of the qualities $\left\{\tau\left(\rho_{12}\right), \tau\left(\rho_{13}\right), \tau\left(\rho_{23}\right)\right\}$ larger than zero, say $\tau\left(\rho_{12}\right)>0$ and $\tau\left(\rho_{13}\right)>0$. According to [20], one can distill two maximal entangled states $\left|\psi_{12}\right\rangle$ and $\left|\psi_{13}\right\rangle$ which belong to $\mathcal{H}_{1} \otimes \mathcal{H}_{2}$ and $\mathcal{H}_{1} \otimes \mathcal{H}_{3}$ respectively. In terms of the result in [26], one can use them to produce a GHZ state.

\section{Conclusions}

We have studied the concurrence for multipartite quantum states and derived an explicit lower bound of the concurrence. This bound can be also served as separability criterion. It detects entanglement of some states better than some separability criteria. For tripartite PPT states the lower bound is zero. The bound also gives sufficient conditions for distilling GHZ states from tripartite states. Moreover it has been shown that there is an interesting relation, similar to the monogamy inequalities and tangle [27], between the lower bound of the concurrence $\tau_{2}$ for bipartite states and $\tau_{3}$ for tripartite states. In addition, our results can be easily generalized to the situation that all the subsystems have different dimensions. By simply neglect the coefficient related to the dimensions, $\frac{d}{2 m(d-1)}$, in the concurrence 
defined in (2), similar results of theorem 2 and 3 hold for systems with different dimensions of subsystems.

Acknowledgments This work is supported by the NSFC 10675086, KZ200810028013, and NKBRPC(2004CB318000).

\section{References}

[1] Nielsen M A, Chuang I L. Quantum Computation and Quantum Information. Cambridge: Cambridge University Press, (2000).

[2] C. H. Bennett, D. P. DiVincenzo and J. A. Smolin, et al. Phys. Rev. A 54, 3824(1996). M. B. Plenio and S. Virmani, Quant. Inf. Comp. 7, 1(2007).

[3] A. Uhlmann Phys. Rev. A 62 032307(2000);

P. Rungta, V. Bužzek, and C. M. Caves, et al. Phys. Rev. A 64, 042315(2001).

[4] S. Albeverio and S. M. Fei, J. Opt. B: Quantum Semiclass Opt, 3, 223-227(2001).

[5] W. K. Wootters, Phys. Rev. Lett. 80, 2245 (1998).

[6] Terhal B M, Vollbrecht K G H, Phys Rev Lett, 85, 2625-2628(2000).

[7] S.M. Fei, J. Jost, X.Q. Li-Jost and G.F. Wang, Phys. Lett. A 310, 333-338(2003).

[8] S.M. Fei and X.Q. Li-Jost, Rep. Math. Phys. 53, 195-210(2004).

[9] S.M. Fei, Z.X. Wang and H. Zhao, Phys. Lett. A 329, 414-419(2004).

[10] P. Rungta and C.M. Caves, Phys Rev A 67, 012307(2003).

[11] F. Mintert, M. Kus, A. Buchleitner, Phys. Rev. Lett. 92, 167902(2004).

[12] K. Chen, S. Albeverio and S.M. Fei, Phys. Rev. Lett. 95, 210501(2005).

[13] K. Chen, S. Albeverio, and S. M. Fei, Phys. Rev. Lett. 95, 040504(2005).

[14] H.P. Breuer, J. Phys. A 39, 11847(2006).

[15] H.P. Breuer, Phys. Rev. Lett. 97, 080501(2006).

[16] J. I. de Vicente, Phys. Rev. A 75, 052320(2007).

[17] C. J. Zhang, Y. S. Zhang, and S. Zhang, et al. Phys. Rev. A 76, 012334(2007).

[18] X. H. Gao, S. M. Fei and K. Wu, Phys. Rev. A 74, 050303(R) (2006). 
[19] E. Gerjuoy, Phys. Rev. A 67, 052308(2003).

[20] Y. C. Ou, H. Fan and S. M. Fei, Phys. Rev. A 78, 012311(2008).

[21] A. Acín, D. Bruss, M. Lewenstein, and A. Sanpera, Phys. Rev. Lett. 87, 040401(2001).

[22] A. S. M. Hassan and P. S. Joag, Quant. Inf. Comp. 8, 0773-0790(2008).

[23] C. H. Bennet, D. P. Divincenzo, Tal Mor, P. W. Shor, J. A. Smolin and B. M. Terhal, Phys. Rev. Lett. 82, 2881(1999).

[24] A. O. Pittenger, and M. H. Rubin, Phys, Rev. A 62, 042306 (2000).

[25] V. Coffman, J. Kundu, and W. K. Wootters, Phys. Rev. A 61, 052306 (2000).

[26] M. Zukowski, A. Zeilinger, M. A. Horne and A. K. Ekert, Phys. Rev. Lett. 71, 4287 (1993).

[27] V. Coffman, J. Kundu, and W. K. Wootters, Phys. Rev. A 61, 052306(2000).

T. J. Osborne and F. Verstraete, Phys. Rev. Lett. 96, 220503(2006).

Y.C. Ou, Phys. Rev. A 75, 034305(2007).

W. Dür, G. Vidal, J. I. Cirac, Phys. Rev. A 62, 062314(2000).

Y.C. Ou, H. Fan, Phys. Rev. A 76, 022320(2007). 